Draft VERSion JANUARY 23, 2018

Preprint typeset using $\mathrm{IAT}_{\mathrm{E}} \mathrm{X}$ style emulateapj v. 5/2/11

\title{
SIMULATIONS OF EARLY BARYONIC STRUCTURE FORMATION WITH STREAM VELOCITY: I. HALO ABUNDANCE
}

\author{
Smadar NaOz ${ }^{1}$, NAOKi Yoshida ${ }^{2}$, Nickolay Y. Gnedin ${ }^{3,4,5}$ \\ Draft version January 23, 2018
}

\begin{abstract}
It has been recently shown that the relative velocity between the dark matter and the baryons $\left(v_{\mathrm{bc}}\right)$ at the time of recombination can affect the structure formation in the early universe (Tseliakhovich \& Hirata 2010). We statistically quantify this effect using large cosmological simulations. We use three different high resolution sets of simulations (with separate transfer functions for baryons and dark matter) that vary in box size, particle number, and the value of the relative velocity between dark matter and baryons. We show that the total number density of halos is suppressed by $\sim 20 \%$ at $z=25$ for $v_{\mathrm{bc}}=1 \sigma_{\mathrm{vbc}}$, where $\sigma_{\mathrm{vbc}}$ is the variance of the relative velocity, while for $v_{\mathrm{bc}}=3.4 \sigma_{\mathrm{vbc}}$ the relative suppression at the same redshift reaches $50 \%$, remaining at or above the $30 \%$ level all the way to $z=11$. We also find high abundance of "empty halos", i.e., halos that have gas fraction below half of the cosmic mean baryonic fraction $\bar{f}_{\mathrm{b}}$. Specifically we find that for $v_{\mathrm{bc}}=1 \sigma_{\mathrm{vbc}}$ all halos below $10^{5} \mathrm{M}_{\odot}$ are empty at $z \geq 19$. The high abundance of empty halos results in significant delay in the formation of gas rich mini-halos and the first galaxies.
\end{abstract}

\section{INTRODUCTION}

Observations of the cosmic microwave background (CMB) show that the Universe at cosmic recombination (redshift $z \sim 10^{3}$ ) was remarkably uniform apart from small spatial fluctuations in the energy density and in the gravitational potential (e.g., Bennett et al. 1996). The primordial inhomogeneities in the density distribution grew over time and eventually led to formation of galaxies, galaxy clusters and large-scale structure. Various physical processes contributed to the growth of perturbations in dark matter and baryons (e.g., Peebles 1980, 1993; Ma \& Bertschinger 1995), which evolved very differently before recombination. As the result, baryon density fluctuations were much smaller than fluctuations in the dark matter density at the time of recombination.

Recently Tseliakhovich \& Hirata (2010) showed that not only the amplitudes of the dark matter and baryon density fluctuations were different but also were their velocities. After recombination, the sound speed of baryons dropped down to thermal velocities, while the dark matter velocity remained unaffected - thus, the relative velocity of the dark matter with respect to the baryons become supersonic. Tseliakhovich \& Hirata (2010) also show that the relative velocity between the dark matter and baryons is coherent on scales of a few mega-parsec and is in the order of $\sim 30 \mathrm{~km} \mathrm{sec}^{-1}$ at the time of cosmic recombination. This relative velocity is often called the "stream velocity" in the literature, and throughout this paper we will use this term. The stream velocity effect was previously overlooked, because the velocity terms are

\footnotetext{
snaoz@northwestern.edu

1 CIERA, Northwestern University, Evanston, IL 60208, USA

2 IPMU, University of Tokyo, 5-1-5 Kashiwanoha, Kashiwa, Chiba 277-8583, Japan

${ }^{3}$ Particle Astrophysics Center, Fermi National Accelerator Laboratory, Batavia, IL 60510, USA

${ }^{4}$ Kavli Institute for Cosmological Physics and Enrico Fermi Institute, The University of Chicago, Chicago, IL 60637 USA

${ }^{5}$ Department of Astronomy \& Astrophysics, The University of Chicago, Chicago, IL 60637 USA
}

actually of the second order in perturbation theory. The stream velocity effect leads to power suppression at mass scales that correspond to masses of the first bound objects in the Universe (e.g., Yoshida et al. 2003a). Using the Press-Schechter (Press \& Schechter 1974) formalism, Tseliakhovich \& Hirata (2010) showed that the number density of halos is suppressed by more than $60 \%$ for halos with $M=10^{6} \mathrm{M}_{\odot}$ at $z=40$. In a subsequent paper, Tseliakhovich et al. (2010) also included the baryonic component following Naoz \& Barkana (2005). They found that the stream velocity also results in much higher minimum mass for a gas rich halo at high redshifts, as compared to the case without the stream velocity (e.g. Naoz \& Barkana 2007).

Tseliakhovich \& Hirata (2010) and Tseliakhovich et al. (2010) findings have prompted several studies on the stream velocity effect in numerical simulations. Stacy et al. (2011) studied the collapse of gas in minihalos and found only a mild delay $\left(\sim 10^{7} \mathrm{yr}\right)$ for the time of collapse at low redshift. They also estimated the difference in redshifts of collapse for the minimum halos that can collapse, with and without the stream velocity effects, and found only minimal effect due to the thermal evolution of the gas collapsing into a minihalo at low redshifts. Their results also show that the delay of the structure formation becomes significant with higher redshift. Maio et al. (2011) examined the influence of the stream velocity on the formation of the smallest bound objects. They showed that the abundance of mini-halos $\left(<10^{7} \mathrm{M}_{\odot}\right)$ is reduced by a few percent and that the baryon fraction in those halos is reduced by somewhat larger amount. Dalal et al. (2010) showed that the characteristic imprint of the stream velocity effect on the power spectrum of the earliest structures can be used to distinguish the effects of minihalos on intergalactic gas before and during reionization. Greif et al. (2011) used high resolution, moving-mesh simulations to study the effect of different initial stream velocities on the virialisation of the gas 
in minihalos; their simulations suggest that the stream velocity substantially delay the onset of gravitational collapse in minihalos. Although all these studies consistently show some delay in the collapse of early gas clouds, a thorough statistical calculation is clearly needed to quantify these results, and, especially, to examine critically the effect on early baryonic structure formation.

In this paper we quantify the suppression of structure formation due to the stream velocity effect by means of cosmological simulations. We use three different sets of high resolution simulations in order to study the stream velocity effect systematically, thus understanding the overall picture (instead of concentrating on specific halos). In a companion paper (Naoz et al. 2011, in prep.) we explore the effect of stream velocity on the gas fraction in halos and compare simulation results to the predictions from linear theory (e.g. Tseliakhovich et al. 2010). We use a set of simulations with different box sizes, particle numbers, and the values for the stream velocity to analyze the suppression of the structure formation as a function of the stream velocity.

We first describe the parameters and initial conditions of our simulations (\$2). We present our results and analysis of the halo mass functions in the simulations in Section 3. Finally we offer a discussion in 8 .

Throughout this paper, we adopt the following cosmological parameters: $\left(\Omega_{\Lambda}, \Omega_{\mathrm{M}}, \Omega_{b}, \mathrm{n}, \sigma_{8}, H_{0}\right)=(0.72,0.28$, $\left.0.046,1,0.82,70 \mathrm{~km} \mathrm{~s}^{-1} \mathrm{Mpc}^{-1}\right)$ (Komatsu et al. 2009).

\section{COSMOLOGICAL SIMULATIONS}

\subsection{Simulation Setup}

We use parallel $N$-body/hydrodynamics solver GADGET-2 (Springel et al. 2001; Springel 2005). We describe the general features of the simulations, which are also summarized in table 1 .

1. The first set, named " $N=256$ ", uses a total of $2 \times 256^{3}$ dark matter and gas particles within a cubic box of 200 comoving kpc on a side. To realize statistically significant number of halos in such a small box, we artificially increase gravitational clustering in the simulation by setting $\sigma_{8}=1.4$. We choose this box size so that a $10^{4} \mathrm{M}_{\odot}$ halo is resolved with $\sim 500$ particles - the value needed to estimate the halo gas fraction reliably (Naoz et al. 2009). The gravitational softening is set to be 40 comoving pc, well below the virial radius of a $10^{4} \mathrm{M}_{\odot}$ halo $(\sim 680$ comoving $\mathrm{pc})$. All the simulations in this set are initialized at $z=199$.

2. The second set, named " $N=512$ ", uses a total of $2 \times 512^{3}$ dark matter and gas particles within a cubic box with the size of $700 \mathrm{kpc}$. In this set we also artificially increase $\sigma_{8}$ to 1.4 . With these parameters, a halo with 500 dark matter particles has a mass of $\sim 5 \times 10^{4} \mathrm{M}_{\odot}$. The softening length is set to be 68 comoving pc. All the simulations in this set are initialized at $z=199$.

3. The final set of simulations uses $2 \times 768^{3}$ dark matter and gas particles (which we name the " $N=$ 768 " set) in a $2 \mathrm{Mpc}$ box, and starts at $z=99$. For these parameters a halo with 500 dark matter
TABLE 1

PARAMETERS OF the Simulations

\begin{tabular}{|c|c|c|c|}
\hline SIM & $\begin{array}{c}v_{\mathrm{bc}, 0} \\
\mathrm{~km} \mathrm{sec}^{-1} \\
\end{array}$ & $\begin{array}{c}\sigma_{\text {vbc }} \text { stream } \\
\text { velocity }\end{array}$ & $\begin{array}{l}\text { Shift in } \\
\text { position }\end{array}$ \\
\hline & 256 runs, & $0.2 \mathrm{Mpc}$ & $z_{i n}=199$ \\
\hline $256_{0}$ & 0 & 0 & No \\
\hline $256_{1 \sigma+p}$ & 5.8 & 1 & Yes $+18.05 \mathrm{kpc} / \mathrm{h}$ \\
\hline $2561.7 \sigma$ & 10 & 1.7 & No \\
\hline $2561.7 \sigma+p$ & 10 & 1.7 & Yes $+30.7 \mathrm{kpc} / \mathrm{h}$ \\
\hline $256_{2.6 \sigma}$ & 15 & 2.6 & No \\
\hline \multirow[t]{2}{*}{$2563.4 \sigma$} & 20 & 3.4 & No \\
\hline & 512 runs, & $0.7 \mathrm{Mpc}$, & $z_{i n}=199$ \\
\hline $512_{0}$ & 0 & 0 & $\mathrm{No}$ \\
\hline $512_{1 \sigma}$ & 5.8 & 1 & No \\
\hline $512_{1.7 \sigma}$ & 10 & 1.7 & No \\
\hline \multirow[t]{2}{*}{$512_{3.4 \sigma}$} & 20 & 3.4 & No \\
\hline & 768 runs, & $2 \mathrm{Mpc}$, & $z_{i n}=99$ \\
\hline $768_{0}$ & 0 & 0 & No \\
\hline $768_{1 \sigma}$ & 3 & 1 & No \\
\hline $7681.7 \sigma$ & 5 & 1.7 & No \\
\hline $768_{3.4 \sigma}$ & 10 & 3.4 & No \\
\hline
\end{tabular}

particle has a mass of $\sim 10^{5} \mathrm{M}_{\odot}$. The softening length is set to be 0.2 comoving kpc. We use the "correct" value of $\sigma_{8}=0.82$ for this simulation set.

In each simulation set, we explore a variety of the values for the stream velocity (see $\$ 2.3$ and table 1).

\subsection{Initial Conditions}

As has been shown by Naoz et al. (2006) and Naoz \& Barkana (2007), setting up initial conditions on the small spatial scales is a delicate issue. High accuracy in initial conditions is crucial for accurately predicting the halo mass function in the lowest mass regime $\left(M \lesssim 10^{7} \mathrm{M}_{\odot}\right)$.

Following NaOz et al. (2010), we generate separate transfer functions for dark matter and baryons as described in Naoz \& Barkana (2005). Ideally, streaming velocities should be realized in the initial conditions in a self-consistent way with the transfer functions that are calculated with streaming effect. However, these transfer functions do not take into account the stream velocity effect, in consistency with the previous calculations that included the stream velocity in simulations. For all runs, glass-like initial conditions were generated using the Zel'dovich approximation. For baryons, we have used a glass file with positions shifted by a random vector, thus removing artificial coupling between nearby dark matter and gas particles (Yoshida et al. 2003b). We note that we have used the same phases for dark matter and baryons in all of our simulations except for those with position shift, see $\$ 2.4$.

\subsection{Stream Velocity in the Simulations}

Tseliakhovich \& Hirata (2010) showed that, while the stream velocity varies in space, its coherence length is quite large, many mega-parsecs. Hence, on scale of our simulation boxes it can be treated as constant bulk motion of baryons with respect to dark matter. We include the effect of stream velocity by adding, at the initial redshift, an additional velocity to the $x$ component of the baryons velocity vector. We test a range of values for the 
stream velocity, which is convenient to quantify in terms of its rms value on small scales, $\sigma_{\mathrm{vbc}}$. Specifically, we test $v_{\mathrm{bc}}=1 \sigma_{\mathrm{vbc}}$ through $v_{\mathrm{bc}}=3.4 \sigma_{\mathrm{vbc}}$ for all the simulations sets (see table 1).

\subsection{Position Shift in the Initial Conditions}

It is customary to set the initial conditions such that the phases of dark matter and baryons are the same, as dictated by the linear perturbation theory. However, because the stream velocity effect is of the second order, it violates the assumption. Namely, since the baryons move in the dark matter reference frame, the respective density perturbations that were co-located at the time of recombination, become separated in space. In other words, the phases of dark matter and baryons at the same spatial location on sufficiently small scales become unrelated.

The relative shift between dark matter and baryons is easily calculated through the geodesic equation,

$$
a \dot{x}=v_{\mathrm{bc}}(a),
$$

where $x$ is the comoving distance, $a$ is the scale factor, and $v_{\mathrm{bc}}(a)$ is the lagrangian velocity of a baryonic fluid element in the dark matter reference frame as a function of the scale factor $a$. Hence

$$
\Delta x_{\mathrm{bc}}(a)=\int_{0}^{t} \frac{v_{\mathrm{bc}}(a)}{a} d t,
$$

where $t$ is the cosmic time at the scale factor $a$. Taking this integral, we find the relative shift in the position between baryons and dark matter at $z_{\text {in }}=199$ is $\Delta x_{\mathrm{bc}}=18.5$ comoving $\mathrm{kpc} / \mathrm{h}$ for $v_{\mathrm{bc}}=1 \sigma_{\mathrm{vbc}}$ and is $\sim 30.7$ comoving $\mathrm{kpc} / \mathrm{h}$ for $v_{\mathrm{bc}}=1.7 \sigma_{\mathrm{vbc}}$. We test the effect of the position shift on two of the $N=256$ runs by adding the above values to the $x$ component of the baryons at the initial redshift $z_{\text {in }}$, thus effectively changing the phases of the baryons relative to dark matter.

As we find below, the relative shift between baryons and dark matter is a small effect, and it does not invalidate the rest of our simulations, where the position shift is neglected.

\subsection{Halo definition}

We locate dark matter halos by running a friends-offriends group-finder algorithm with a linking parameter of 0.2 (only for the dark matter component). We use the identified particle groups to find the center of mass of each halo. After the center is located, we calculate density profiles of dark matter and baryons separately, assuming a spherical halo and using 2000 radial bins between $r_{\min }=0 \mathrm{kpc}$ and $r_{\max }=20 \mathrm{kpc}$. Using the density profiles, we find the virial radius $r_{v i r}$ at which the total overdensity is 200 times the mean background density, and compute the mass and the gas fraction of each halo within that radius.

Recently, More et al. (2011) showed that halos identified by the friends-of-friends algorithm enclose an average overdensity that is substantially larger than 200 and its specific value depends on the halo concentration. In our approach we use the friends-of-friends algorithm only for finding the center of mass of a halo, and compute the actual halo mass using the spherical overdensity of 200.

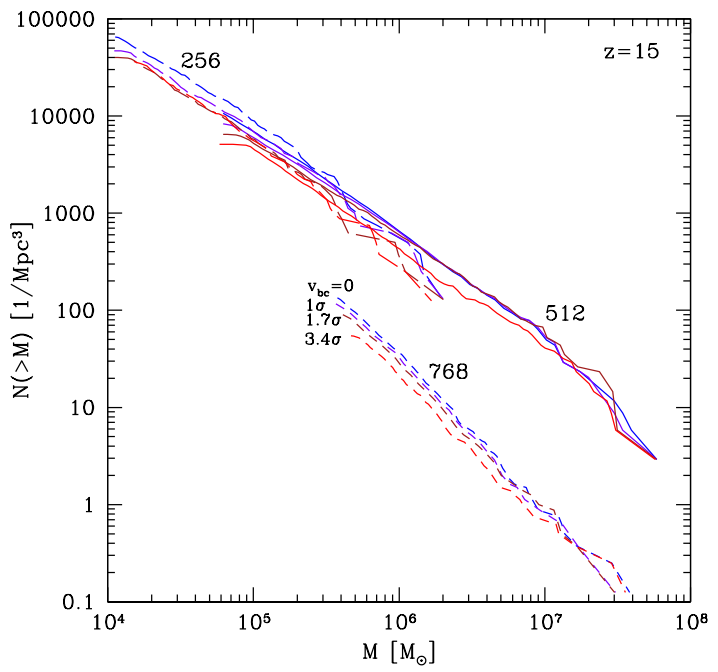

FIG. 1.- Cumulative halo mass function for different simulations and valus of $v_{b c}$ at $z=15$. We show the $N=256$ (long dashed lines), $N=512$ (solid lines), and $N=768$ (short dashed lines). We consider the values for the stream velocity of $v_{b c}=0, v_{\mathrm{bc}}=1 \sigma_{\mathrm{vbc}}$, $v_{\mathrm{bc}}=1.7 \sigma_{\mathrm{vbc}}$, and $v_{\mathrm{bc}}=3.4 \sigma_{\mathrm{vbc}}$, from top to bottom in each sets of lines.

We only retain halos that contain at least 500 dark matter particles within their virial radii. The choice allows us to estimate halo masses to about $15 \%$ precision (Trenti et al. 2010) and to estimate halo gas fractions reliably to a similar level of accuracy (Naoz et al. 2009).

\section{RESULTS}

\subsection{Suppression of the Halo Mass Function}

We first consider all the simulations that do not include the relative position shift between baryons and dark matter (i.e., all runs except $N=256_{1 \sigma+p}$ and $N=256_{1.7 \sigma+p}$ ). Tseliakhovich \& Hirata (2010) calculated the number densities of collapsed halos with and without the stream velocity, and illustrated the stream velocity effect on the abundance of small halos. Specifically, based on the Press-Schechter formalism, they showed that the number density of haloes with the rms stream velocity $N_{\mathrm{vbc}}(>M)$ is suppressed by more than $60 \%$ at the mass scale of $M \sim 10^{6} \mathrm{M}_{\odot}$ in comparison to the case with no stream velocity $\left(N_{0}(>M)\right)$.

In Figure 11 we show the halo mass functions at $z=$ 15 for all our simulations. Indeed, as can be seen, the halo number density is a strong function of the stream velocity, resulting in substantial suppression of the halo mass function for typical values of the stream velocity 6

Following Tseliakhovich \& Hirata (2010), we quantify the suppression of the halo mass function as

$$
\Delta_{\mathrm{v}}=\frac{N_{\mathrm{vbc}}(>M)-N_{0}(>M)}{N_{0}(>M)} .
$$

A more relevant quantity is, perhaps, the abundance of halos that contain substantial amount of gas. It is such halos that may host first stars and first supernovae, and also serve as sinks of ionizing radiation during reioniza-

6 The lower halo mass function for the $N=768$ runs is the result of our simulation setup; $N=768$ have much lower $\sigma_{8}$ than other simulation sets. 

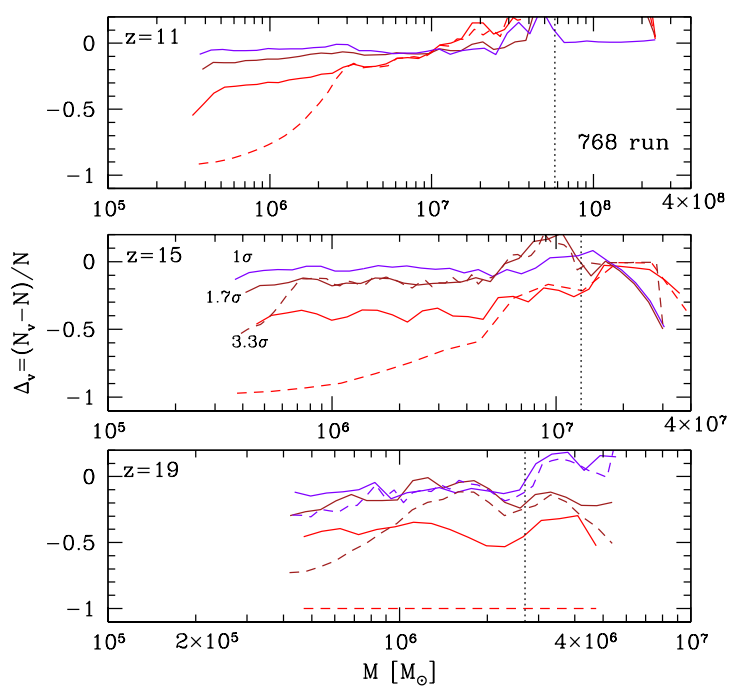

FIG. 2.- Effect of the stream velocity on the cumulative halo mass function for the $N=768$ runs, for redshifts of (from bottom to top panels) 19,15 , and 11 . The relative difference for all halos $\left(\Delta_{\mathrm{V}}\right)$ is shown with the solid lines, while dashed lines show the relative difference for non-empty halos $\left(\Delta_{\mathrm{v}, f_{g}}\right)$, for various values of the stream velocity $v_{\mathrm{bc}}=1 \sigma_{\mathrm{vbc}}, v_{\mathrm{bc}}=1.7 \sigma_{\mathrm{vbc}}$, and $v_{\mathrm{bc}}=3.4 \sigma_{\mathrm{vbc}}$ (purple, brown and red lines respectively). Note that $\Delta_{\mathrm{v}, f_{g}}$ for $v_{\mathrm{bc}}=3.4 \sigma_{\mathrm{vbc}}$ at $z=19$ is exactly -1 (i.e., there are no halos with gas fraction above $\bar{f}_{\mathrm{b}} / 2$ in that simulation). The opposite is true for $v_{\mathrm{bc}}=1 \sigma_{\mathrm{vbc}}$ at $z=15$ and $z=11$ - there are no empty halos in those runs. The dotted vertical line in each panel marks the mass at which the halo number density is $5 \mathrm{kpc}^{-3}$.

tion. Therefore, we also introduce

$$
\Delta_{\mathrm{v}, f_{g}}=\frac{N_{\mathrm{vbc}}\left(>M, f_{g}>\bar{f}_{\mathrm{b}} / 2\right)-N_{0}\left(>M, f_{g}>\bar{f}_{\mathrm{b}} / 2\right)}{N_{0}\left(>M, f_{g}>\bar{f}_{\mathrm{b}} / 2\right)}
$$

as a relative difference between the mass functions of halos that contain more gas than one half of the cosmic mean baryon fraction $\left(\bar{f}_{\mathrm{b}}\right)$ in simulations with and without the stream velocity, $N_{\mathrm{vbc}}\left(>M, f_{g}>\bar{f}_{\mathrm{b}} / 2\right)$ and $N_{0}\left(>M, f_{g}>\bar{f}_{\mathrm{b}} / 2\right)$ respectively.

We would call halos with gas fractions less then $\bar{f}_{\mathrm{b}} / 2$ "empty halos", although, admittedly, this definition is somewhat arbitrary and a different gas fraction threshold could also be considered.

First we show in Figure 2 the relative suppression of halo mass function due to the stream velocity for all and for non-empty halos only $\left(\Delta_{\mathrm{v}}\right.$ and $\Delta_{\mathrm{v}, f_{g}}$; solid and dashed curves respectively) for the $N=768$ set of simulations. At $z=19$ the suppression of the total number density of halos is about $50 \%$ for $10^{6} \mathrm{M}_{\odot}$ for $v_{\mathrm{bc}}=3.4 \sigma_{\mathrm{vbc}}$ and $\sim 15 \%$ for $v_{\mathrm{bc}}=1 \sigma_{\mathrm{vbc}}$. As time goes by, the effect of the stream velocity diminishes, but remains clearly visible even at the lowest redshift we consider, $z=11$, still present.

Perhaps the most interesting feature shown in Figure 2 is the high abundance of empty halos. At $z=19$, more than $20 \%$ of the halos below $10^{6} \mathrm{M}_{\odot}$ are empty for $v_{\mathrm{bc}}=1.7 \sigma_{\mathrm{vbc}}$ and all of the halos in the simulation are empty for $v_{\mathrm{bc}}=3.4 \sigma_{\mathrm{vbc}}$. These empty halos lay above the minimum cooling mass $7\left(\sim 3 \times 10^{5} \mathrm{M}_{\odot}\right.$,

7 We note that we in the simulations presented in the paper we included only adiabatic cooling.

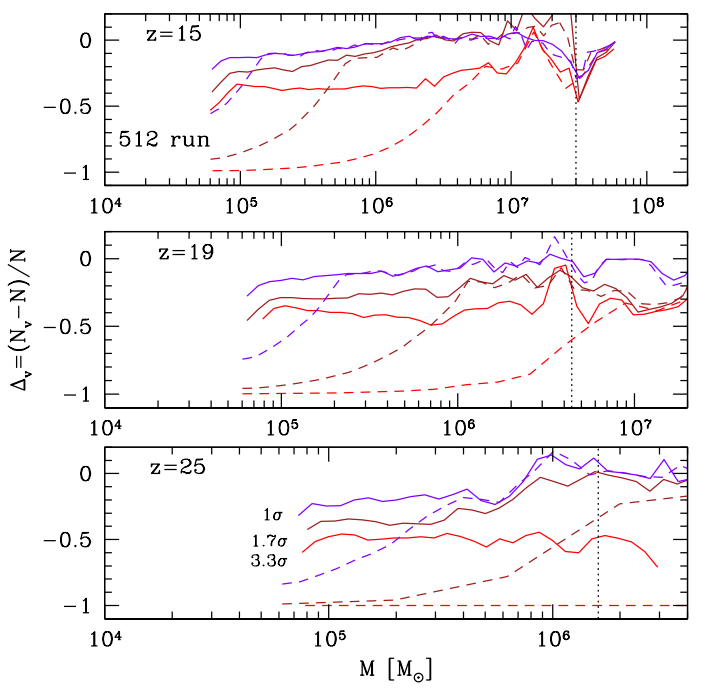

Fig. 3.- Same as Figure 2 for the $N=512$ runs at redshifts (from bottom to top panels) 25, 19, and 15 .

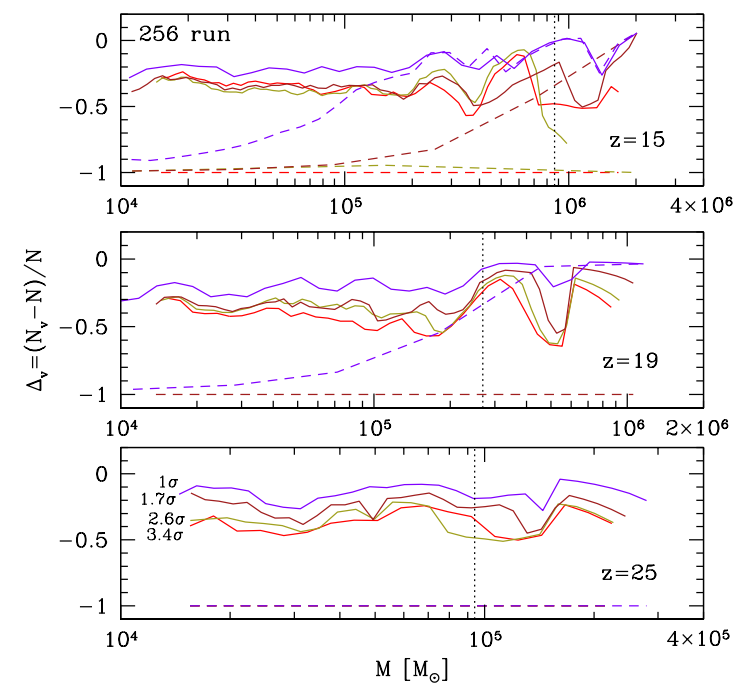

FIG. 4.- Same as Figure 2 for the $N=256$ runs at redshifts (from bottom to top panels) 25, 19, and 15 and for 4 values of the stream velocity $v_{\mathrm{bc}}=1 \sigma_{\mathrm{vbc}}, v_{\mathrm{bc}}=1.7 \sigma_{\mathrm{vbc}}, v_{\mathrm{bc}}=2.6 \sigma_{\mathrm{vbc}}$, and $v_{\mathrm{bc}}=3.4 \sigma_{\mathrm{vbc}}$ (purple, brown, green and red lines respectively).

e.g., Trenti \& Stiavelli 2009). Thus, we conclude that, in patches of the universe where the stream velocity is high, the formation of the first stars and galaxies is delayed. This behavior is consistent with the $N=512$ simulation set (Figure 3), where, for $v_{\mathrm{bc}}=3.4 \sigma_{\mathrm{vbc}}$, most of the halos below $\sim 10^{6} \mathrm{M}_{\odot}$ are empty of baryons at $z=19$, and all of the halos in the simulation are empty at $z=25$. Furthermore, for $v_{\mathrm{bc}}=1.7 \sigma_{\mathrm{vbc}}$, more than $50 \%$ of the halos below $\sim 4 \times 10^{5} \mathrm{M}_{\odot}$ are empty for $z \geq 19$.

\subsection{Saturation of the Halo Mass Function Suppression in the Low Mass Limit}

As expected, larger values for the stream velocity result in stronger suppression of the halo mass function. However, in the $N=256$ simulation set the effect clearly saturates for high values of $v_{\mathrm{bc}}$ (see Figure 4). In order to explore the nature of this saturation further, we com- 


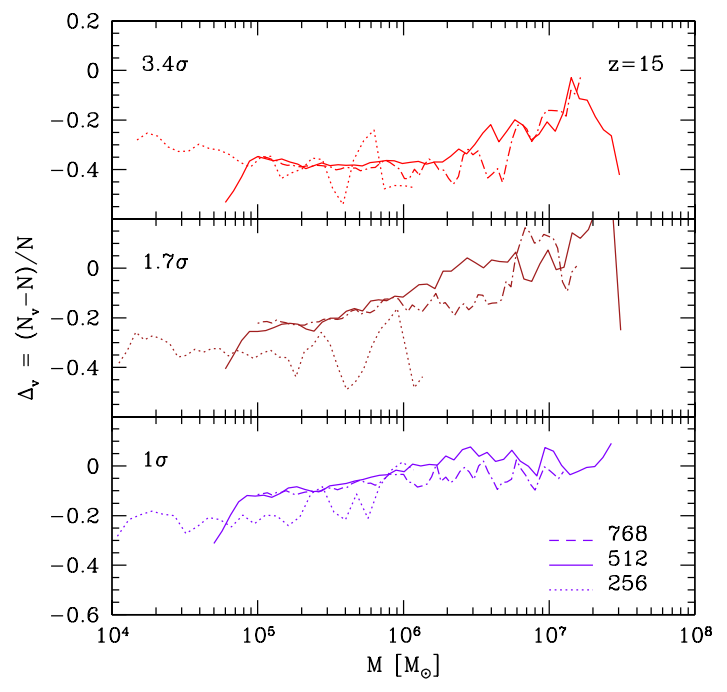

Fig. 5.- Halo mass function suppression factor $\Delta_{\mathrm{v}}$ at $z=15$ for all our simulation sets for different values of the stream velocity (from bottom to top) of $v_{\mathrm{bc}}=1 \sigma_{\mathrm{vbc}}, 1.7 \sigma_{\mathrm{vbc}}$, and $3.4 \sigma_{\mathrm{vbc}}$. In all panels $N=768, N=512$, and $N=256$ simulation sets are represented by dashed, solid, and dotted lines respectively. All 3 simulations sets are reasonably consistent with each other within the cosmic variance. Note that to check consistency with somewhat lower mass values, we have lowered the threshold for the number of particles per halo to 300 particles per halos only for the $N=768$ runs and only in order to generate this figure.

pare $\Delta_{\mathrm{v}}$ at $z=15$ (the latest epoch that our $N=256$ runs can be continued to) between different simulation sets in Figure [5]

Overall, the agreement between all three simulation sets is reasonable 8 ; $N=256$ set suffers from poor statistics for $M \gtrsim 10^{5} \mathrm{M}_{\odot}$, so the agreement between that series and two other sets is somewhat worse. Nevertheless, the effect of saturation for low masses is clearly visible for all values of $v_{\mathrm{bc}}$.

The reason why $\Delta_{\mathrm{V}}$ as a function of halo mass is expected to saturate at the low mass limit is understood as follows. For halo mass of $10^{5} \mathrm{M}_{\odot}$ the escape velocity is about $0.77 \mathrm{~km} \mathrm{sec}^{-1}$, while the stream velocity for $v_{\mathrm{bc}}=3.4 \sigma_{\mathrm{vbc}}$ at $z=15$ is $1.6 \mathrm{~km} \mathrm{sec}^{-1}$ and at $z=25$ is $2.6 \mathrm{~km} \mathrm{sec}^{-1}$. Thus, it is not surprising that halos below $10^{5} \mathrm{M}_{\odot}$ are empty in that redshift range - the stream velocity is simply much larger then the halo escape velocity, so the dark matter halo is unable to accrete any gas. Hence, such a halo will have only about $\left(1-\bar{f}_{\mathrm{b}}\right)$ of the mass it would have had if $v_{\mathrm{bc}}=0$. Since the cumulative mass function $N(>M) \propto M^{-1}$ in this mass regime, the loss of baryons by low mass halos results in $\bar{f}_{\mathrm{b}} \approx 0.17$ reduction in the halo mass function.

At larger masses the interaction between the baryons moving with the highly supersonic velocity and the dark matter halo becomes more complex. For example, in the $v_{\mathrm{bc}}=3.4 \sigma_{\mathrm{vbc}}$ case the mass function is smaller by $\sim 40 \%$ at $10^{5} \mathrm{M}_{\odot} \lesssim M \lesssim 3 \times 10^{6} \mathrm{M}_{\odot}$. This magnitude of the effect cannot be explained by the loss of baryons alone; some non-negligible fraction of dark matter has to be lost by a halo as well. Note that the simulations sets $N=512$ and $N=768$ have different $\sigma_{8}$, but agree well

\footnotetext{
${ }^{8}$ Note that this agreement is present through all the simulations despite the different $\sigma_{8}$ assumed.
}

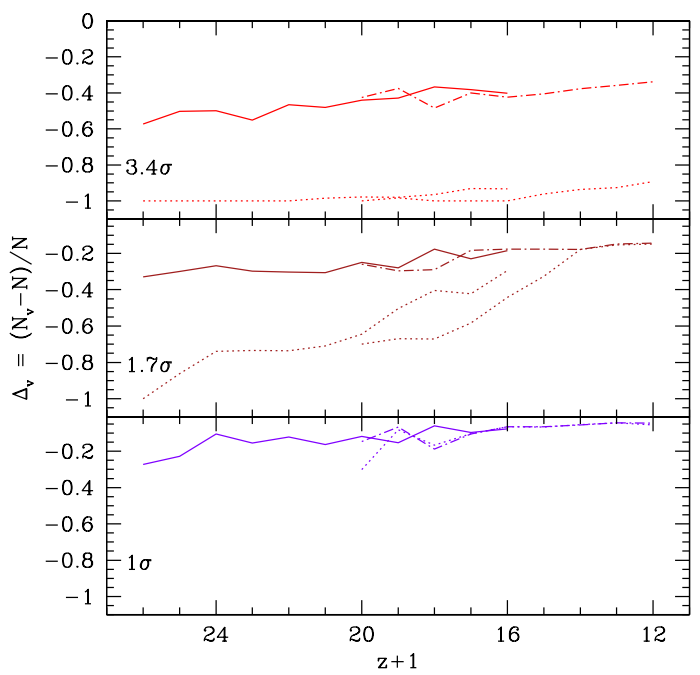

FIG. 6.- Halo mass function suppression factor $\Delta_{\mathrm{V}}$ as a function of time for $5 \times 10^{5} \mathrm{M}_{\odot}$ halos. In this figure we only show $N=768$ (dot-dashed curves) and $N=512$ (solid curves) simulation sets. We also show the suppression factor for non-empty halos, $\Delta_{\mathrm{v}, \mathrm{f}_{\mathrm{g}}}$, with dotted lines. We consider $v_{\mathrm{bc}}=1 \sigma_{\mathrm{vbc}}, v_{\mathrm{bc}}=1.7 \sigma_{\mathrm{vbc}}$, and $v_{\mathrm{bc}}=3.4 \sigma_{\mathrm{vbc}}$ from bottom to top panels respectively.

in the magnitude of the halo mass function suppression. Hence, these results suggest that the suppression effect is largely due to non-trivial non-linear interaction of the baryonic flow and the dark matter halo.

In Figure 5 we consider the time evolution of the halo mass function suppression factor for a halo mass that lays safely above the molecular hydrogen cooling mass (e.g., Trenti \& Stiavelli 2009). We choose a value of $5 \times$ $10^{5} \mathrm{M}_{\odot}$, which is well resolved and statistically reliably represented in both $N=768$ and $N=512$ simulation sets. This comparison suggests significant delay in the formation epoch of gas rich halos, which, in principle, could have formed stars. We find that for $v_{\mathrm{bc}}=1 \sigma_{\mathrm{vbc}}$ the suppression is modest, in agreement with previous studies (Maio et al. 2011; Stacy et al. 2011). However, for larger values of the stream velocity $\left(v_{\mathrm{bc}}=1.7 \sigma_{\mathrm{vbc}}\right.$ and $\left.v_{\mathrm{bc}}=3.4 \sigma_{\mathrm{vbc}}\right)$ we find a significant delay and large abundance of empty halos.

\subsection{Position Shift}

As we discussed in $\$ 2.4$ the stream velocity also causes the baryons to shift in position relative to dark mater. We have tested this effect in two of the $N=256$ simulation runs. In Figure 7 we show the relative suppression of halo formation comparing between the cases with and without the position shift (thin and thick curves respectively). By $z=15$ the relative shift in the positions is erased; at earlier times the effect is noticeable, but it never becomes the dominant effect in the change in the halo mass function induced by the stream velocity.

\subsection{Suppression of the Clumping Factor}

The baryonic clumping factor plays an important role in the penetration and escape of radiation from an inhomogeneous medium and thus the effects of the stream velocity also depicted in the suppression of the clumping factor (e.g. Barkana \& Loeb 2001). Adopting Springel \& Hernquist (2003) for estimating the clumping 

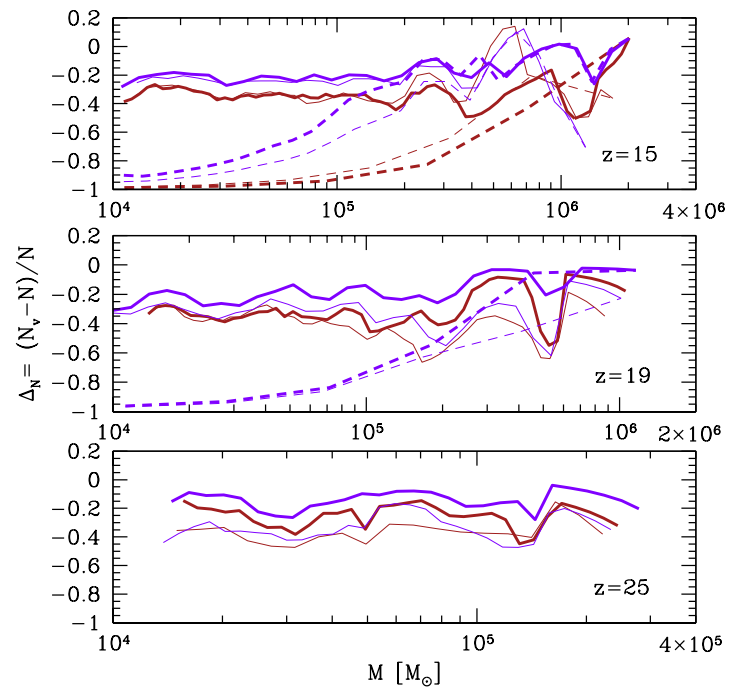

FIG. 7.- Halo mass function suppression factors $\Delta_{\mathrm{V}}$ (solid curves) and $\Delta_{\mathrm{v}, f_{g}}$ (dashed curves) for $v_{\mathrm{bc}}=1 \sigma_{\mathrm{vbc}}$ and $v_{\mathrm{bc}}=$ $1.7 \sigma_{\mathrm{vbc}}$ cases (purple and brown curves respectively). Thin curves represent simulations that properly account for the position shift $\left(256_{1 \sigma+p}\right.$ and $256_{1.7 \sigma+p}$ runs), while thick curves show our fiducial approach, without adding a position shift to the initial conditions (i.e., initializing the simulation with the same phases for the baryons and dark matter, $256_{1 \sigma_{\mathrm{vbc}}}$ and $256_{1.7 \sigma}$ ). As one can see, the relative shift in the positions of baryons and dark matter makes only a modest correction.

TABLE 2

Fitting Parameters

\begin{tabular}{lcccc}
$v_{\mathrm{bc}}$ & $a_{1}$ & $a_{2}$ & $a_{3}$ & $a_{4}$ \\
\hline \hline 0 & $415.1 \pm 66.4$ & $-0.32 \pm 0.01$ & $0.06 \pm 0.12$ & $0.13 \pm 0.09$ \\
$1 \sigma_{\mathrm{vbc}}$ & $393.8 \pm 42.3$ & $-0.33 \pm 0.01$ & $0.05 \pm 0.07$ & $0.15 \pm 0.08$ \\
$1.7 \sigma_{\mathrm{vbc}}$ & $439.4 \pm 54.4$ & $-0.35 \pm 0.01$ & $0.12 \pm 0.15$ & $0.1 \pm 0.06$ \\
$3.4 \sigma_{\mathrm{vbc}}$ & $607.1 \pm 75.7$ & $-0.41 \pm 0.01$ & $0.55 \pm 0.27$ & $0.04 \pm 0.02$ \\
\hline
\end{tabular}

factor $C$ from $\mathrm{SPH}$ simulations we define

$$
C=\frac{\sum_{i} m_{b, i} \rho_{i}\left(<\Delta \rho_{c}\right)^{-1} \sum_{j} m_{b, j} \rho_{j}\left(<\Delta \rho_{c}\right)}{\left(\sum_{k} m_{b, k}\right)^{2}},
$$

where $m_{b}$ is the mass of the baryon particles in our simulations, $\rho_{c}$ is the critical density in the Universe, and the summation is over all gas particles.

Following Maio et al. (2006) we sum over all gas particles whose density $\rho_{i}$ is smaller than a given threshold $\Delta \rho_{c}$. Thus, we avoid numerical artifacts that are produced by gas particles belonging to collapsed objects which would artificially increase the value of $C$ due to their high density. In Figure 8 we show the clumping factor for the $N=768$ run. We choose to show the results of only this set of runs because the clumping factor depends on the assumed initial fluctuation amplitudes. For this run the gas particle mass is $m_{b}=225.5 \mathrm{M}_{\odot}$ for all SPH particles. In Figure 8, bottom panel, we show the clumping factor for overdensity of $\Delta=200$, and we also test $\Delta=500$ (dotted lines). Note, that we omit the

\footnotetext{
${ }^{9}$ Unlike the variables $\Delta_{v}$ and $\Delta_{v, f_{g}}$ the clumping factor is very sensitive to the assumed initial fluctuation amplitudes (because essentially we count the number of clumps, which is larger for large $\left.\sigma_{8}\right)$
}

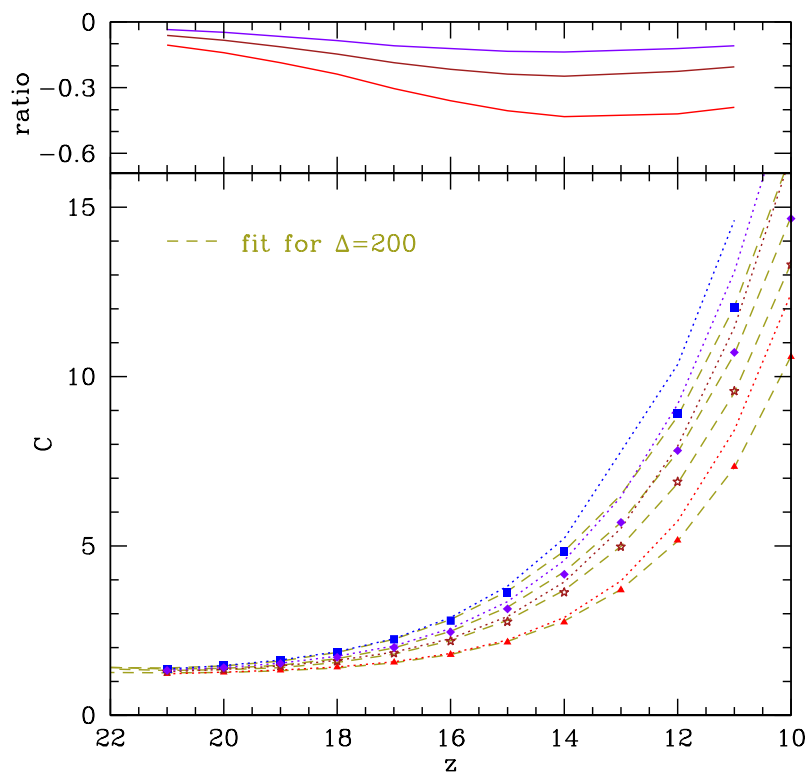

Fig. 8. - The clumping factor as a function of redshift. In the bottom panel we consider the clumping factor evolution for $v_{\mathrm{bc}}=$ $0, v_{\mathrm{bc}}=1 \sigma_{\mathrm{vbc}}, v_{\mathrm{bc}}=1.7 \sigma_{\mathrm{vbc}}$, and $v_{\mathrm{bc}}=3.4 \sigma_{\mathrm{vbc}}$ (blue,purple, brown and red lines respectively), choosing $\Delta=200$. We show in dashed lines the resulted fit to equation (6); see table 2 for the fit parameters. In the top panel we consider the suppression ratio $C_{\mathrm{vbc}} / C_{\mathrm{vbc}=0}-1$ (see text) for overdensity case of $\Delta=200$ (we use the same color code as in previous figures). Note that the results for $z=13$ and $z=10$ of the run $768_{0}$ are missing due to computer failure.

$\Delta=100$ lines from the figure, to reduce the clutter, this choice gives a typical $1 \%$ reduction of the clumping factor at $z=14$ and about $15 \%$ at $z=10$ (for all $v_{\mathrm{bc}}$ cases). We also consider (top panel) the relative suppression ratio $C_{\mathrm{vbc}} / C_{\mathrm{vbc}=0}-1$, where $C_{\mathrm{vbc}}$ is the clumping factor for the case with stream velocity and $C_{\mathrm{vbc}=0}$ is for the case without the stream velocity effect.

The growth of the clumping factor as a function of time is quite regular with a behavior, at high redshift, that resembles exponential asymptotic decay. We assume an exponential behavior and find a simple fit in the form of:

$$
C(z)=a_{1} e^{a_{2} z}+a_{3} \exp ^{a_{4} z} .
$$

We summarize the fit results (for $\Delta=200$ for different values of $v_{\mathrm{bc}}$ ) in table 2 .

As shown in Figure 8 the stream velocity suppresses the clumping of baryon especially at lower redshift. At high redshift the clumping is similar and indeed low in all cases. However, as time goes by, the clumpiness in the case without stream velocity increases dramatically, while it is smaller by about $50 \%$ for $v_{\mathrm{bc}}=3.4 \sigma_{\mathrm{vbc}}$. Figure 8 also shows that the suppression reaches a saturation at $z=14-12$ and below this redshift we find smaller differences.

\section{DISCUSSION}

We have used three-dimensional hydrodynamical simulations to investigate the effects of stream velocity on halo mass function in the early universe (in a companion paper Naoz et al. 2011, we study the effect on the gas fraction and filtering mass). Tseliakhovich \& Hirata (2010) showed recently, within the frame work of linear theory, 
that the initial velocity difference between baryons and dark matter after recombination significantly suppresses the halo mass function at small scales.

We found that the total halo mass function as a function of mass is significantly suppressed in all of our simulation sets (see Figures 24). Summarizing Figures 2 6, we find that for a range of halos between $5 \times 10^{4}-5 \times 10^{5} \mathrm{M}_{\odot}$ the suppression of the number density of halos at $z=25$ is $\sim 20 \%$ for $v_{\mathrm{bc}}=1 \sigma_{\mathrm{vbc}}, \sim 40 \%$ for $v_{\mathrm{bc}}=1.7 \sigma_{\mathrm{vbc}}$, and $\sim 55 \%$ for $v_{\mathrm{bc}}=3.4 \sigma_{\mathrm{vbc}}$. As time goes on, the suppression of the halo mass function due to the stream velocity diminishes with a varied rate - for example, by $z=15$ (the lowest redshift we consider in this paper), for $v_{\mathrm{bc}}=1 \sigma_{\mathrm{vbc}}$ the halo mass function approaches the case with no stream velocity (i.e., no suppression), while in the $v_{\mathrm{bc}}=3.4 \sigma_{\mathrm{vbc}}$ case the suppression factor remains at $\sim 30 \%$.

Our most significant result is the high fraction of halos that are almost entirely devoid of gas. We have found that for the extreme values of the stream velocity we consider $\left(v_{\mathrm{bc}}=3.4 \sigma_{\mathrm{vbc}}\right)$, the gas simply does not accrete on the dark matter halos - almost all halos below $10^{6} \mathrm{M}_{\odot}$ throughout all of our simulation runs, are empty. For more typical values of the stream velocity $\left(v_{\mathrm{bc}}=1 \sigma_{\mathrm{vbc}}\right)$ most of the empty halos are smaller than about $10^{5} \mathrm{M}_{\odot}$ at $z \leq 19$. Thus, we expect significant delay in the formation of the first generation of galaxies; in our companion paper Naoz et al. (2011, in prep.) we study the mass scales of halos that still retain their gas.

It was suggested in the literature that gas rich halos and minihalos may play an important role in cosmic reionization, and that they can produce distinct 21-cm signatures (Kuhlen et al. (2006); Shapiro et al. (2006); Naoz \& Barkana (2008) but see Furlanetto \& Oh $(2006))$. Minihalos can block ionizing radiation and induce an overall delay in the initial progress of reionization (e.g., Shapiro \& Giroux 1987; Barkana \& Loeb 2002; Iliev et al. 2003; Shapiro et al. 2004; Iliev et al. 2005; McQuinn et al. 2007). However, our results here (and in our companion paper Naoz et al. 2011, in prep.) suggest that at high redshift the stream velocity effect results in a high abundance of empty halos halos below $\sim 10^{5} \mathrm{M}_{\odot}$. Thus, if reionization started sufficiently early (Yoshida et al. 2007), in patches of the universe where the stream velocity is large, there are fewer gas rich halos that can absorb ionizing photons. Hence, in these patches the delay of the reionization caused by minihalos may be less than in regions that happen to have a small value of the stream velocity. The feature is clearly quantified by using the gas clumping factor. The variable measure the clumpiness of baryonic structure outside of bound objects. We found that it is very sensitive to stream velocity effect (see Figure 8). Therefore, not only the formation of the first generations of galaxies may be affected by the stream velocity effect, but also the reionization process may proceed differently in regions with very different stream velocities.

We note that recently Bittner \& Loeb (2011) showed that if the ionizing sources were primarily in halos dominated by atomic cooling (corresponding to $\gtrsim 10^{8} \mathrm{M}_{\odot}$ ), then the effect on reionization is negligible. However, for sources at high redshift, in halos dominated by molecular hydrogen the effect on the evolution of the ionizing front may be significant.

\section{ACKNOWLEDGMENTS}

We thank Avi Loeb, Rennan Barkana, Andrey Kravtsov, Neal Dalal, Will Farr and Dmitriy Tseliakhovich for useful discussions. We also thank Yoram Lithwick for the use of this allocation time on the computer cluster Quest. This research was supported in part through the computational resources and staff contributions provided by Information Technology at Northwestern University as part of its shared cluster program, Quest. SN acknowledges support from a Gruber Foundation Fellowship and from the National Post Doctoral Award Program for Advancing Women in Science (Weizmann Institute of Science). This work was supported in part by the DOE at Fermilab, by the NSF grant AST0908063, and by the NASA grant NNX-09AJ54G. NY acknowledges financial support from from the Grant-inAid for Scientific Research (S) 20674003 by Japan Society for the Promotion of Science.

\section{REFERENCES}

Barkana, R., \& Loeb, A. 2001, Phys. Rep., 349, 125, arXiv:astro-ph/0010468

- 2002, ApJ, 578, 1, arXiv:astro-ph/0204139

Bennett, C. L. et al. 1996, ApJ, 464, L1+ arXiv:astro-ph/9601067

Bittner, J. M., \& Loeb, A. 2011, ArXiv e-prints, 1110.4659

Dalal, N., Pen, U., \& Seljak, U. 2010, JCAP, 11, 7, 1009.4704

Furlanetto, S. R., \& Oh, S. P. 2006, ApJ, 652, 849, arXiv:astro-ph/0604080

Greif, T., White, S., Klessen, R., \& Springel, V. 2011, ArXiv e-prints, 1101.5493

Iliev, I. T., Scannapieco, E., Martel, H., \& Shapiro, P. R. 2003, MNRAS, 341, 81, arXiv:astro-ph/0209216

Iliev, I. T., Scannapieco, E., \& Shapiro, P. R. 2005, ApJ, 624, 491, arXiv:astro-ph/0411035

Komatsu, E. et al. 2009, ApJS, 180, 330, 0803.0547

Kuhlen, M., Madau, P., \& Montgomery, R. 2006, ApJ, 637, L1, arXiv:astro-ph/0510814

Ma, C.-P., \& Bertschinger, E. 1995, ApJ, 455, 7 , arXiv:astro-ph/9506072

Maio, U., Dolag, K., Meneghetti, M., Moscardini, L., Yoshida, N., Baccigalupi, C., Bartelmann, M., \& Perrotta, F. 2006, MNRAS, 373, 869, arXiv:astro-ph/0607409
Maio, U., Koopmans, L. V. E., \& Ciardi, B. 2011, MNRAS, $\mathrm{L} 197+, 1011.4006$

McQuinn, M., Lidz, A., Zahn, O., Dutta, S., Hernquist, L., \& Zaldarriaga, M. 2007, MNRAS, 377, 1043,

arXiv:astro-ph/0610094

More, S., Kravtsov, A. V., Dalal, N., \& Gottlöber, S. 2011, ApJS, 195, 4, 1103.0005

Naoz, S., \& Barkana, R. 2005, MNRAS, 362, 1047,

arXiv:astro-ph/0503196

. 2007, MNRAS, 377, 667, arXiv:astro-ph/0612004

. 2008, MNRAS, 385, L63, 0707.3146

Naoz, S., Barkana, R., \& Mesinger, A. 2009, MNRAS, 399, 369, 0906.0349

Naoz, S., Noter, S., \& Barkana, R. 2006, MNRAS, 373, L98, arXiv:astro-ph/0604050

Naoz, S., Yoshida, N., \& Barkana, R. 2010, ArXiv e-prints, 1009.0945

Naoz, S., Yoshida, N., \& Gnedin, N. 2011, ArXiv e-prints

Peebles, P. J. E. 1980, The large-scale structure of the universe, ed. Peebles, P. J. E.

. 1993, Principles of Physical Cosmology, ed. Peebles, P. J. E.

Press, W. H., \& Schechter, P. 1974, ApJ, 187, 425 
Shapiro, P. R., Ahn, K., Alvarez, M. A., Iliev, I. T., Martel, H., \& Ryu, D. 2006, ApJ, 646, 681, arXiv:astro-ph/0512516

Shapiro, P. R., \& Giroux, M. L. 1987, ApJ, 321, L107

Shapiro, P. R., Iliev, I. T., \& Raga, A. C. 2004, MNRAS, 348, 753, arXiv:astro-ph/0307266

Springel, V. 2005, MNRAS, 364, 1105, arXiv:astro-ph/0505010

Springel, V., \& Hernquist, L. 2003, MNRAS, 339, 312, arXiv:astro-ph/0206395

Springel, V., Yoshida, N., \& White, S. D. M. 2001, Nature, 6, 79, arXiv:astro-ph/0003162

Stacy, A., Bromm, V., \& Loeb, A. 2011, ApJ, 730, 728, 1011.4512

Trenti, M., Smith, B. D., Hallman, E. J., Skillman, S. W., \& Shull, J. M. 2010, ApJ, 711, 1198, 1001.5037
Trenti, M., \& Stiavelli, M. 2009, ApJ, 694, 879, 0901.0711

Tseliakhovich, D., Barkana, R., \& Hirata, C. 2010, ArXiv e-prints, 1012.2574

Tseliakhovich, D., \& Hirata, C. 2010, Phys. Rev. D, 82, 083520, 1005.2416

Yoshida, N., Abel, T., Hernquist, L., \& Sugiyama, N. 2003a, ApJ, 592, 645, arXiv:astro-ph/0301645

Yoshida, N., Omukai, K., \& Hernquist, L. 2007, ApJ, 667, L117, 0706.3597

Yoshida, N., Sugiyama, N., \& Hernquist, L. 2003b, MNRAS, 344, 481, arXiv:astro-ph/0305210 


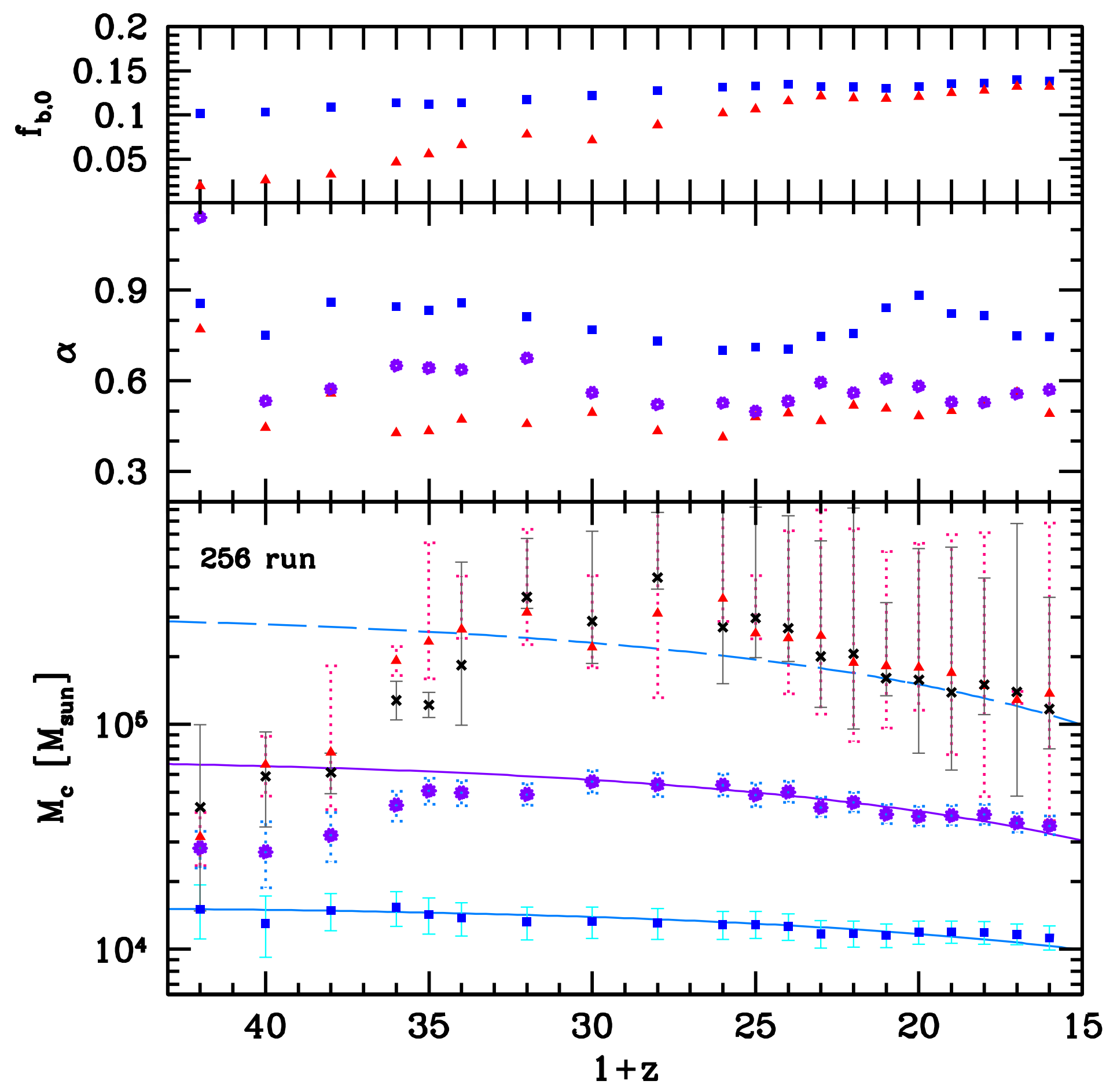




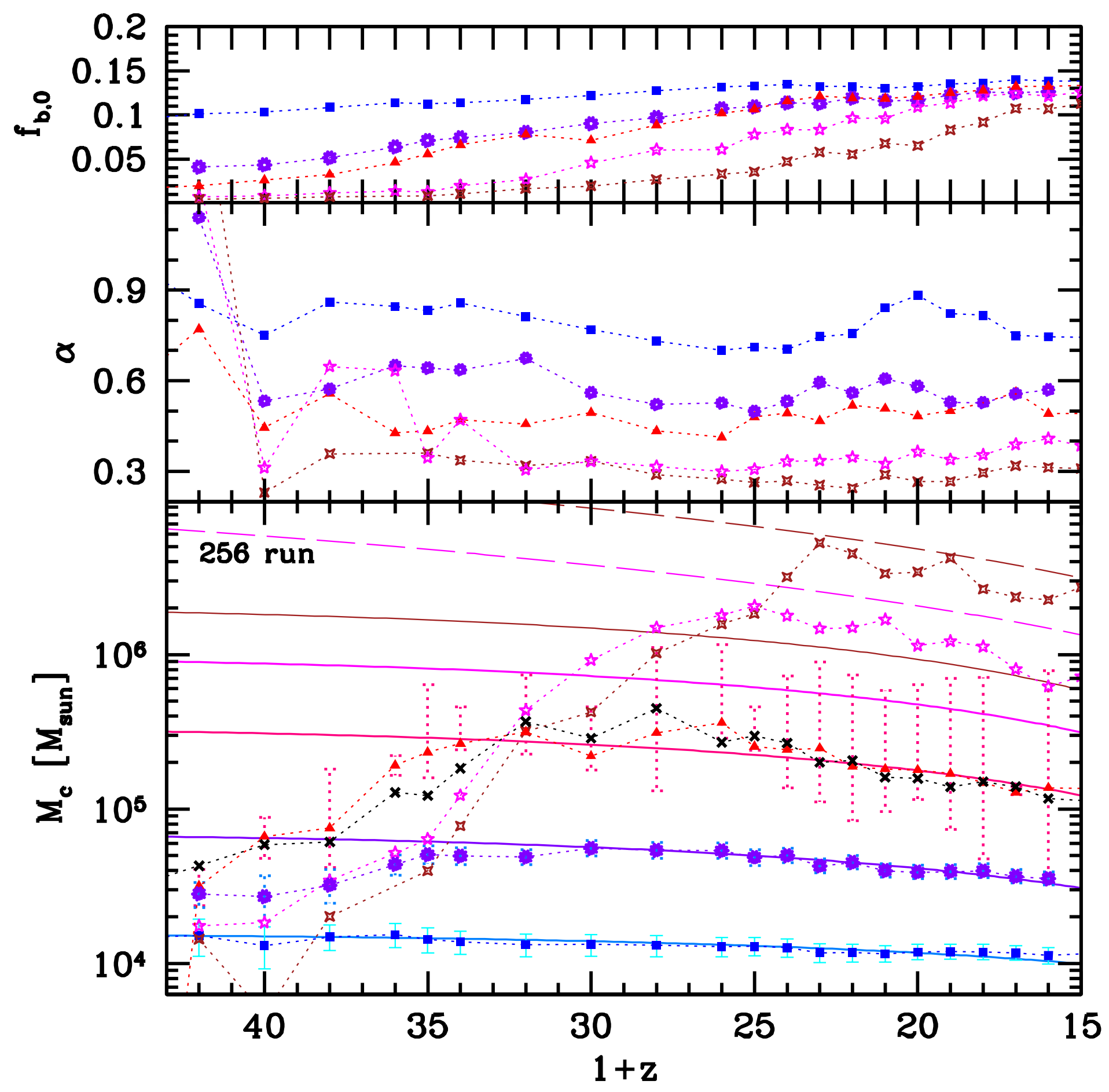




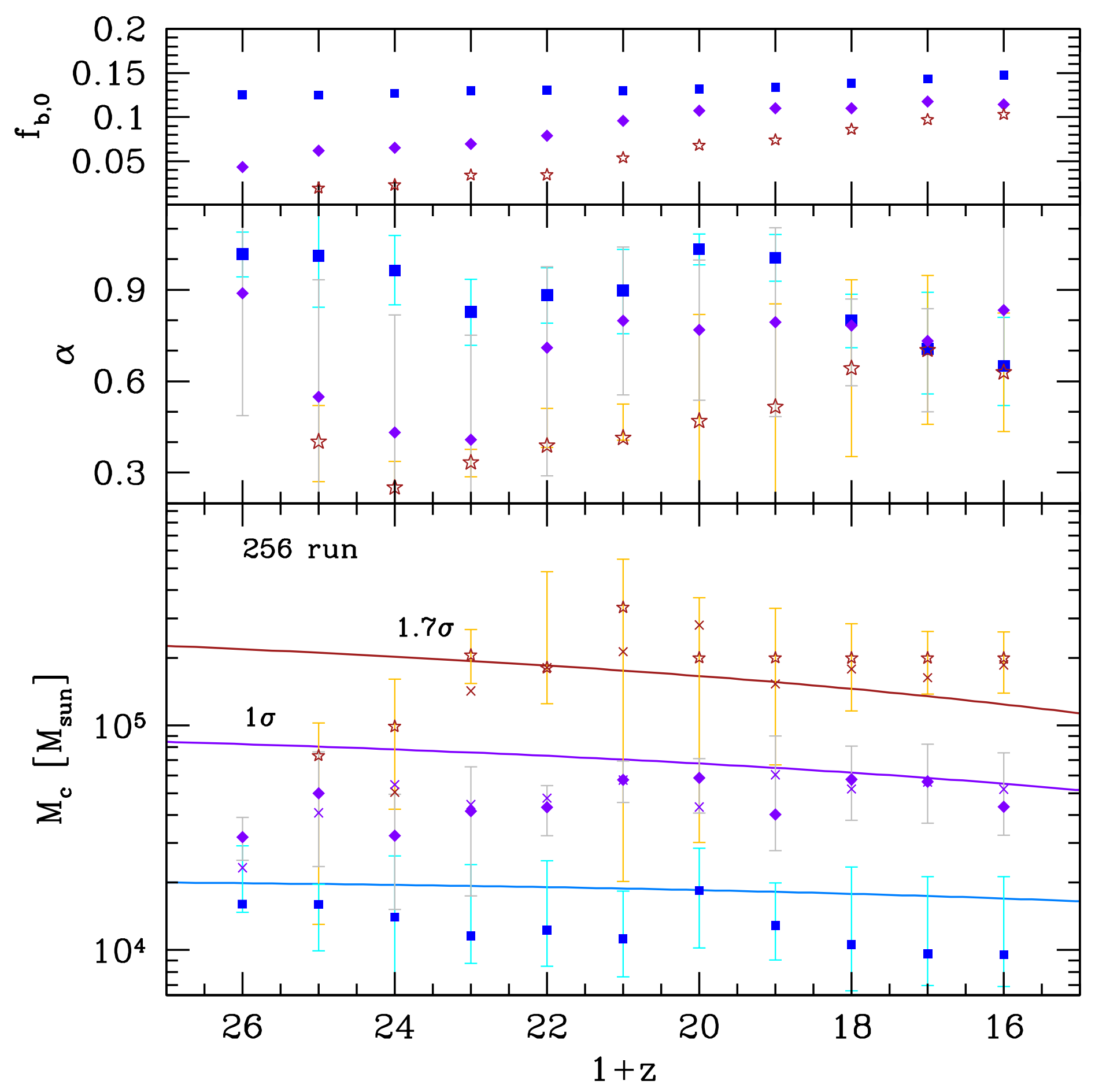




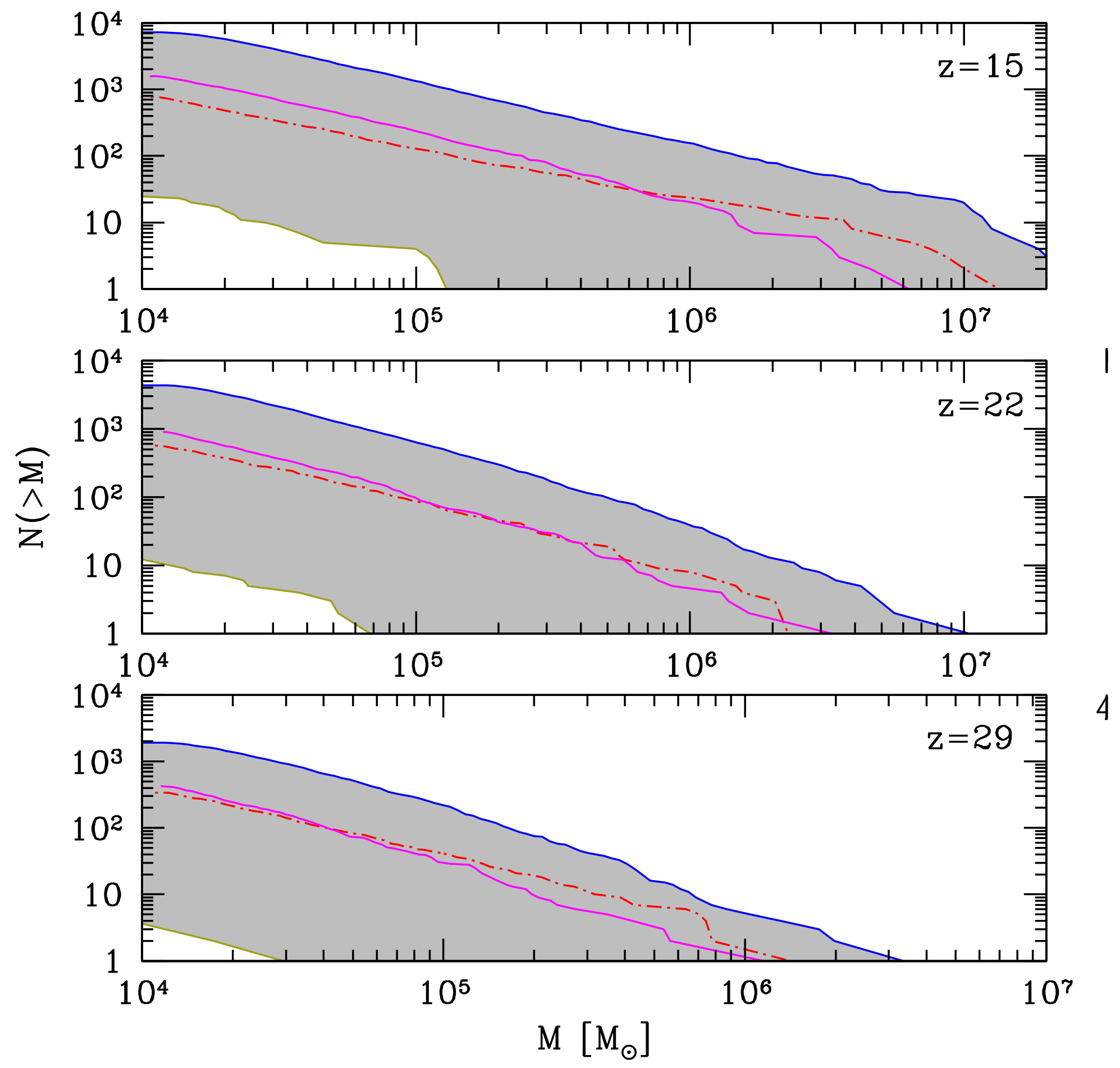




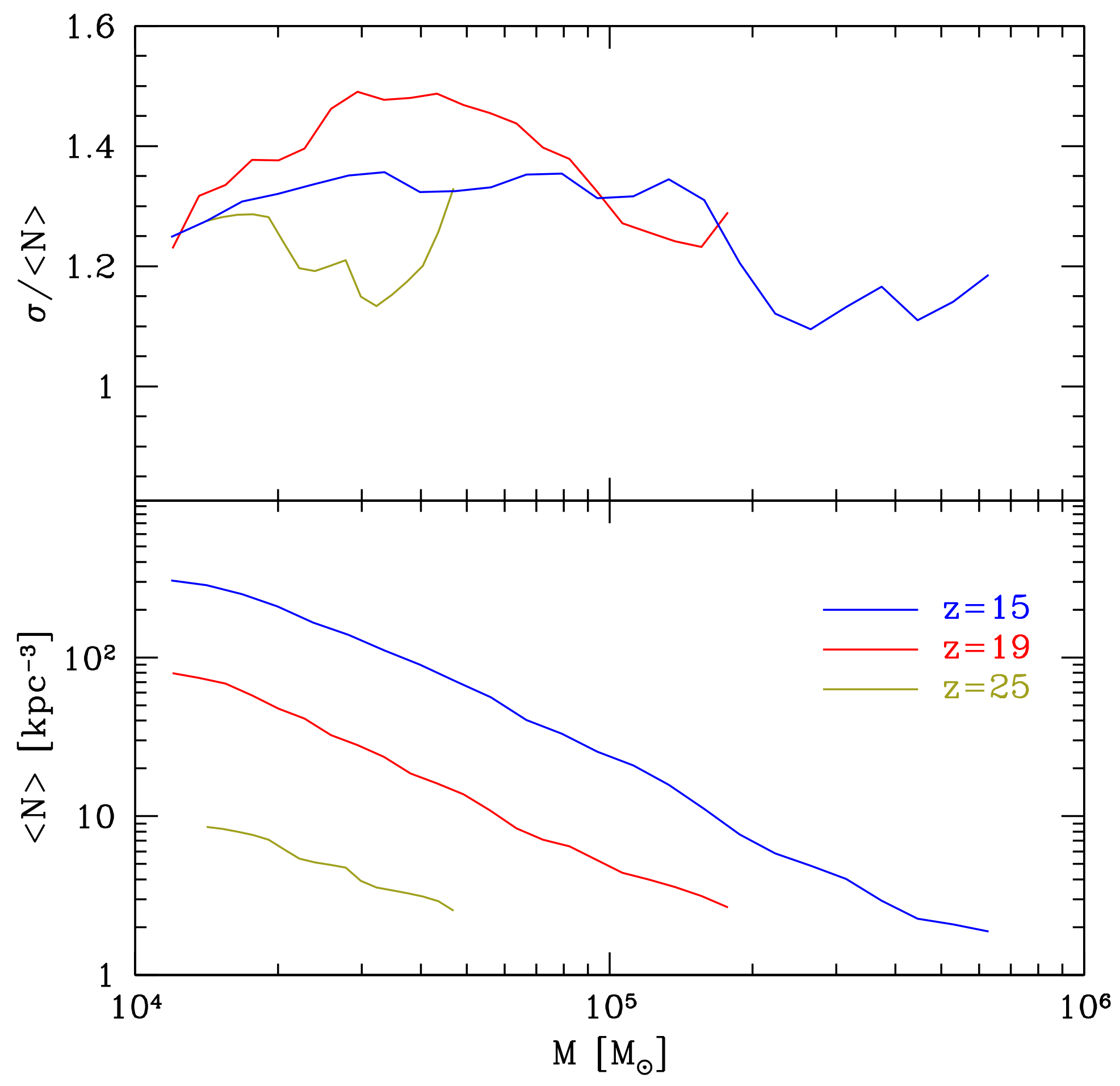



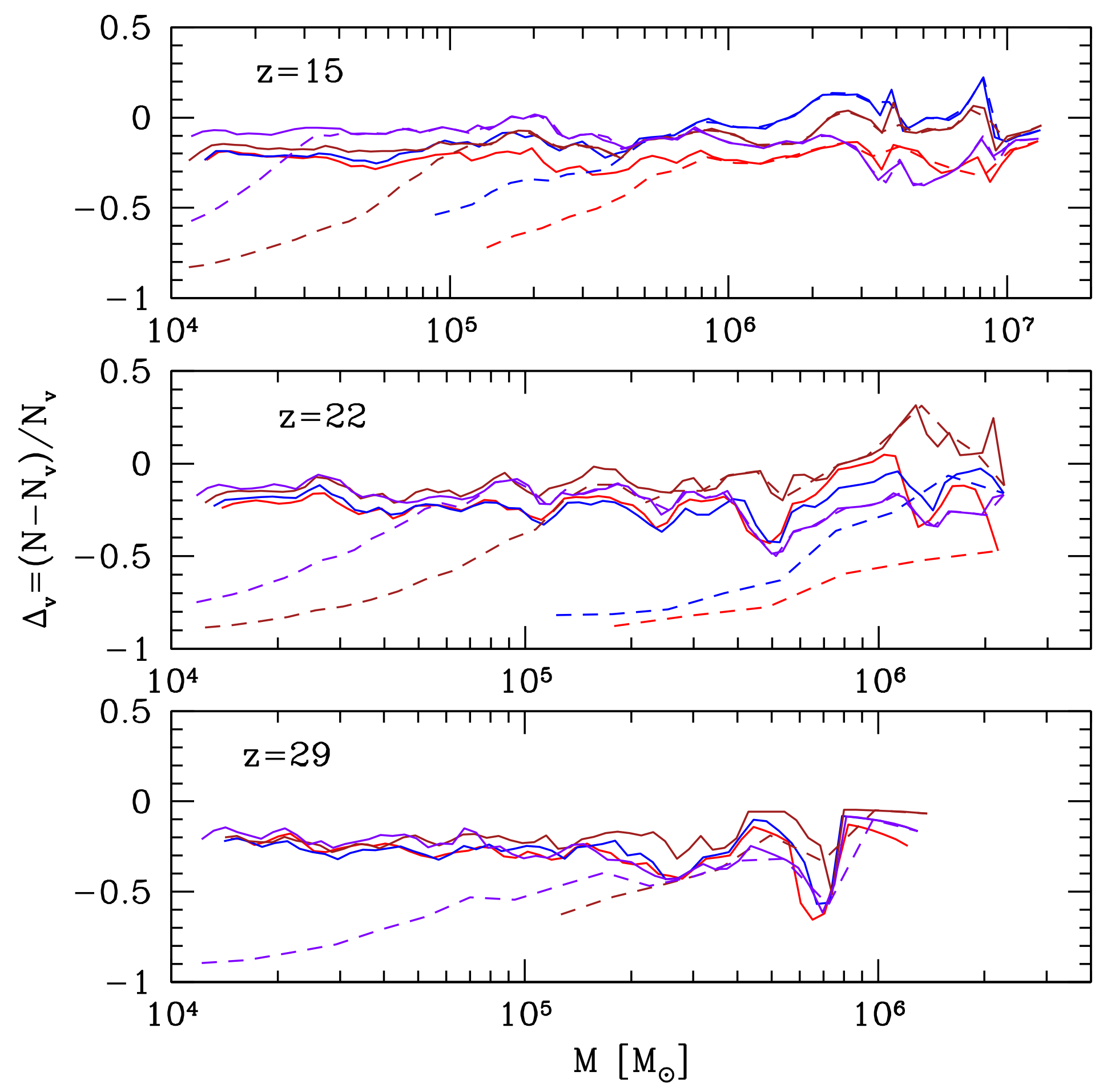


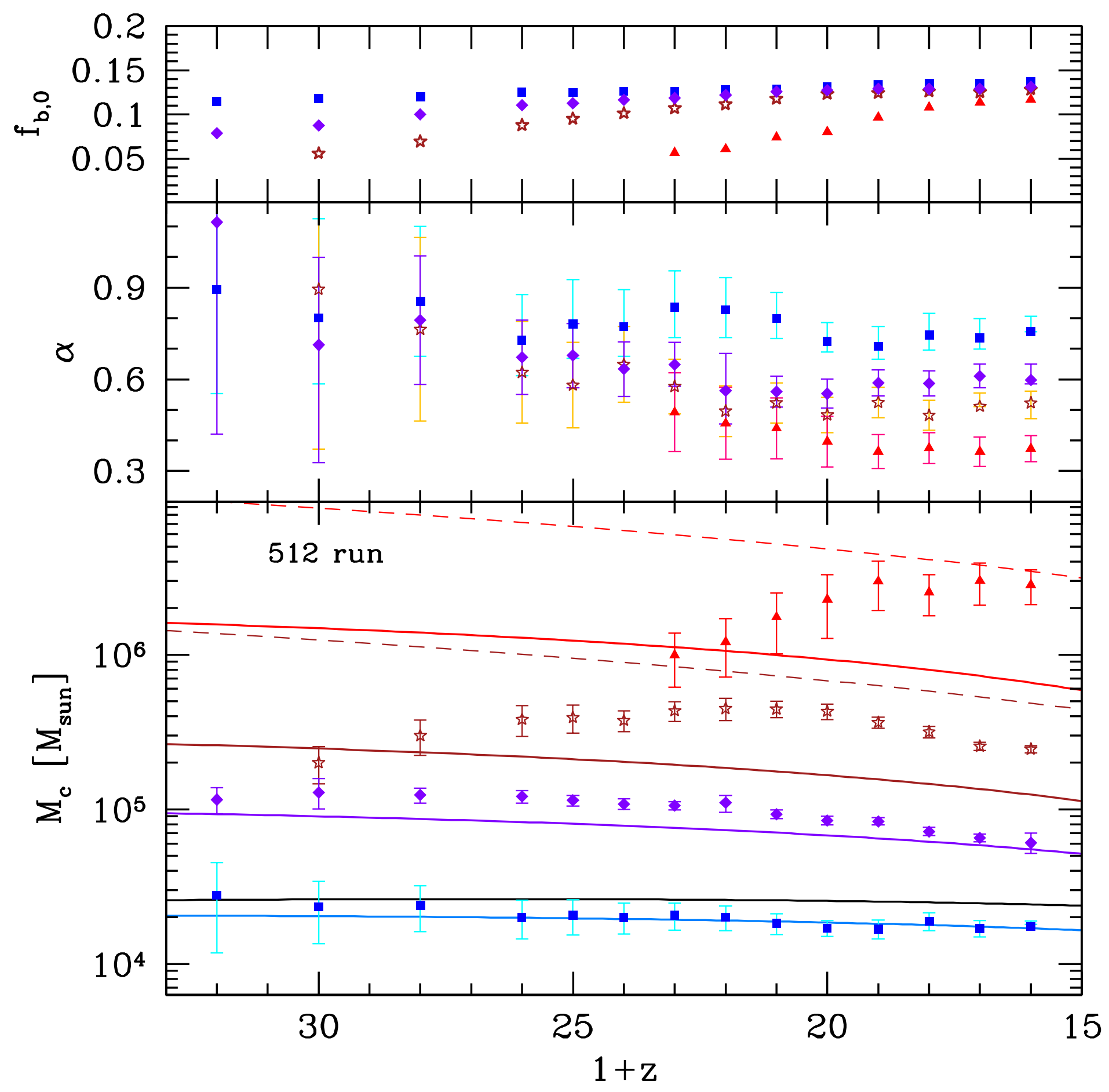




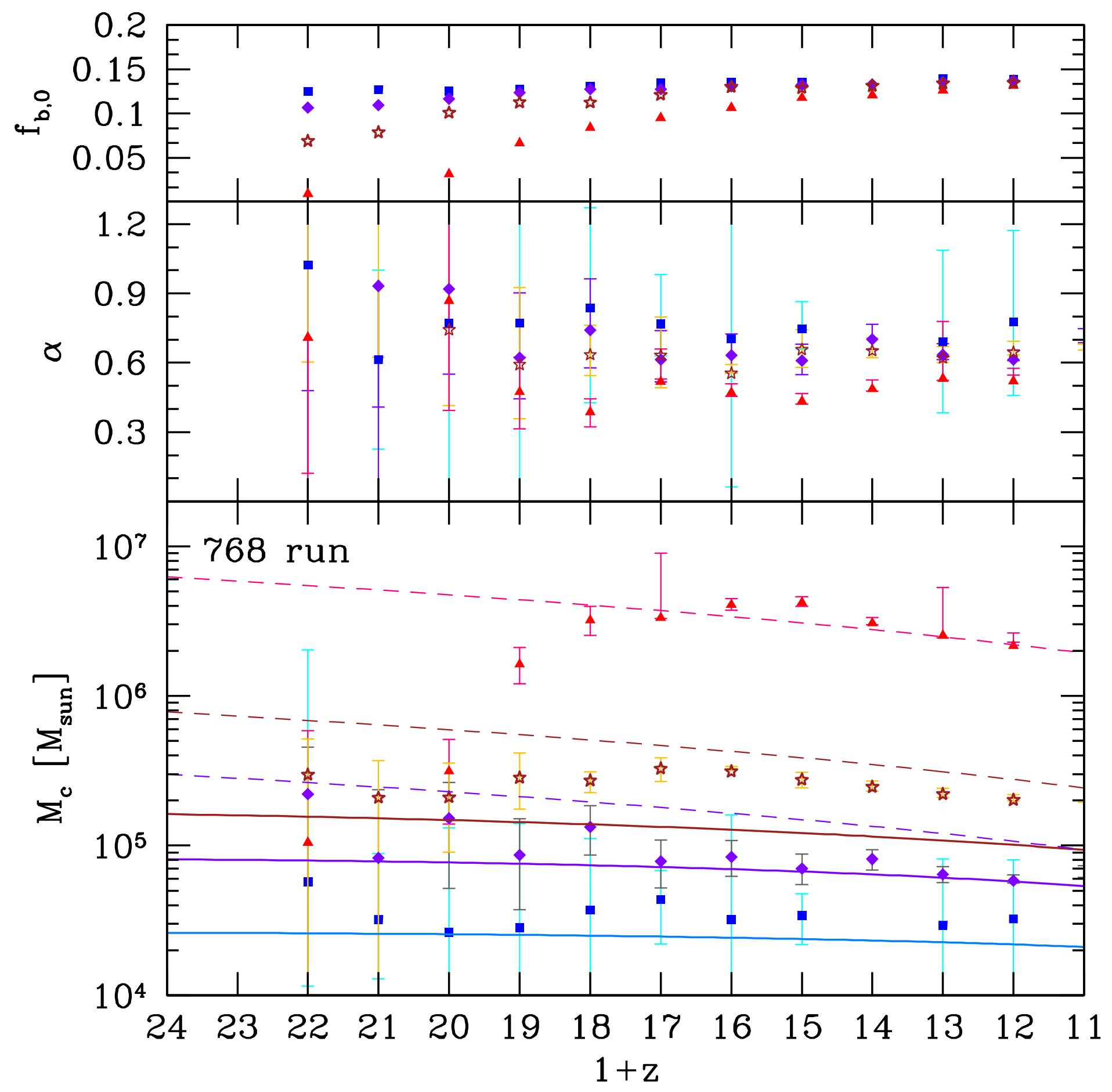




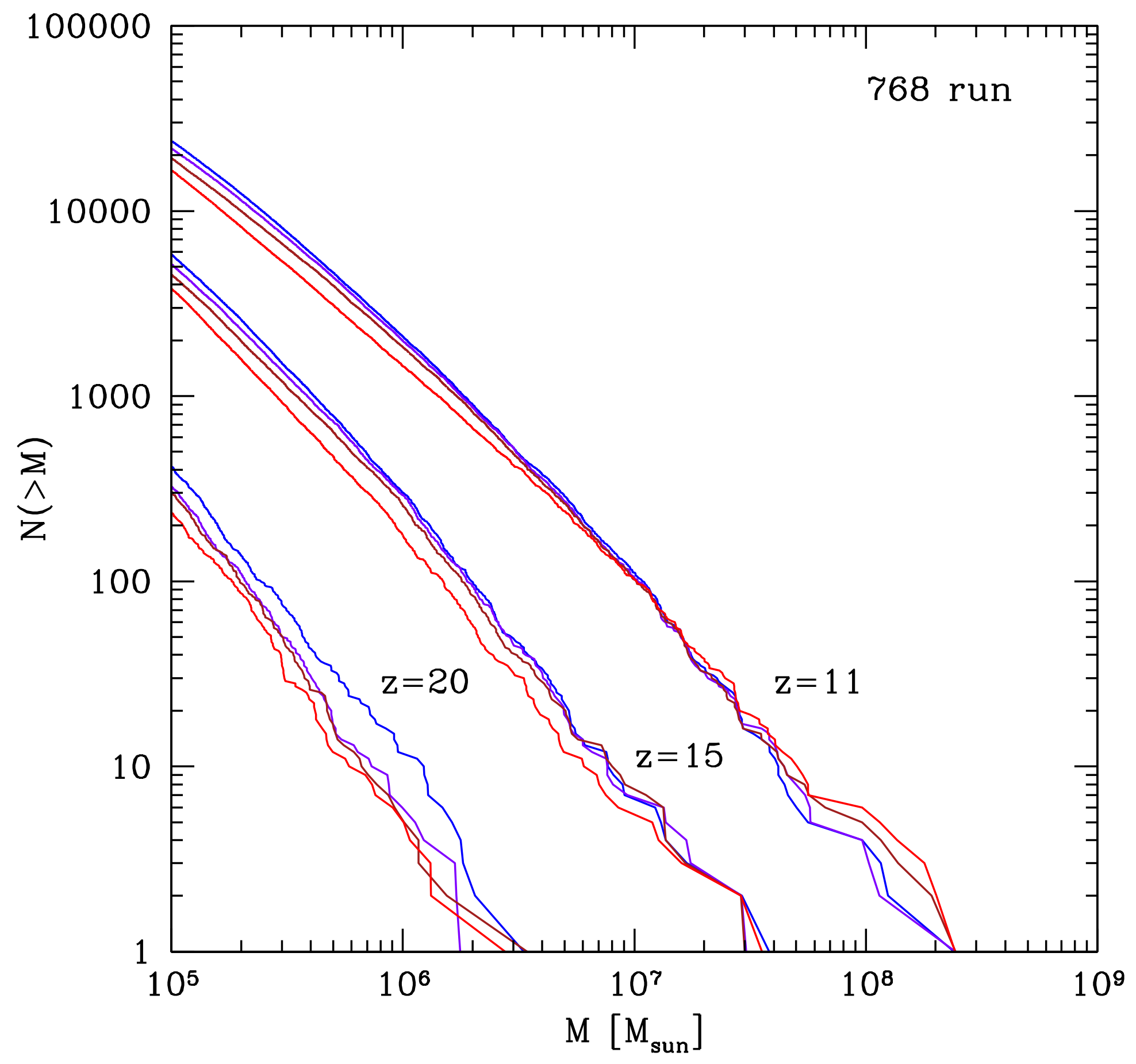




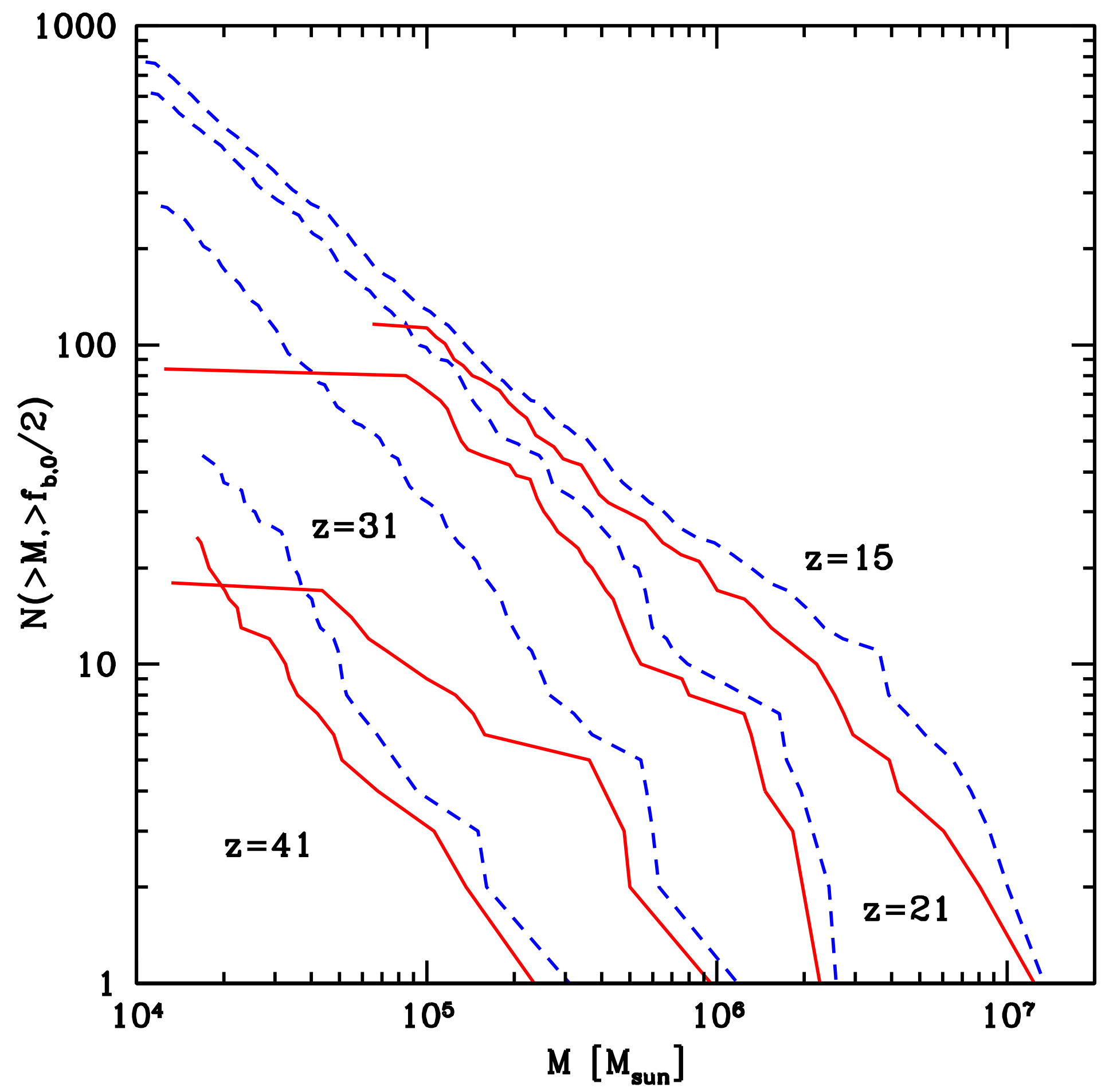




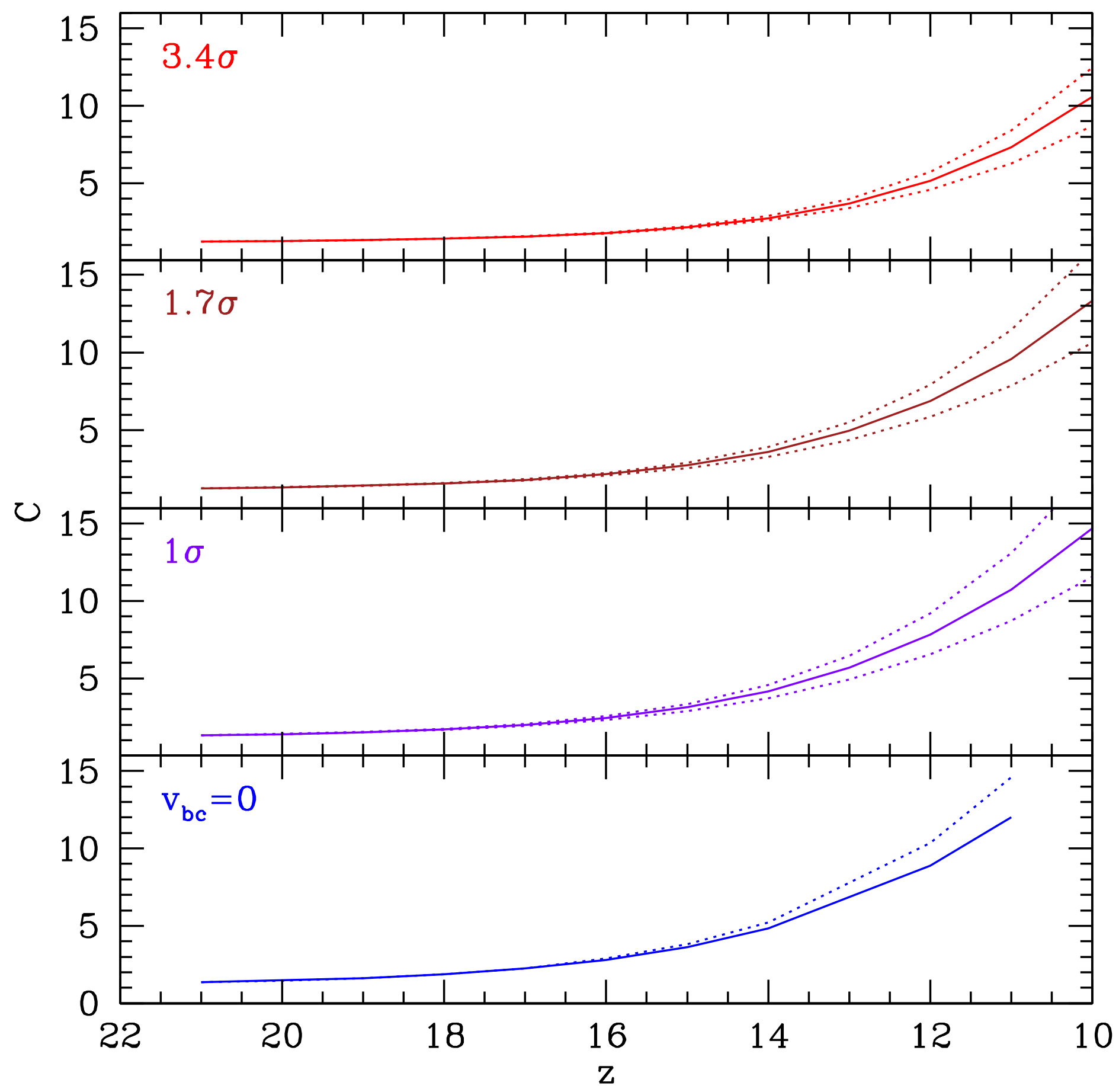




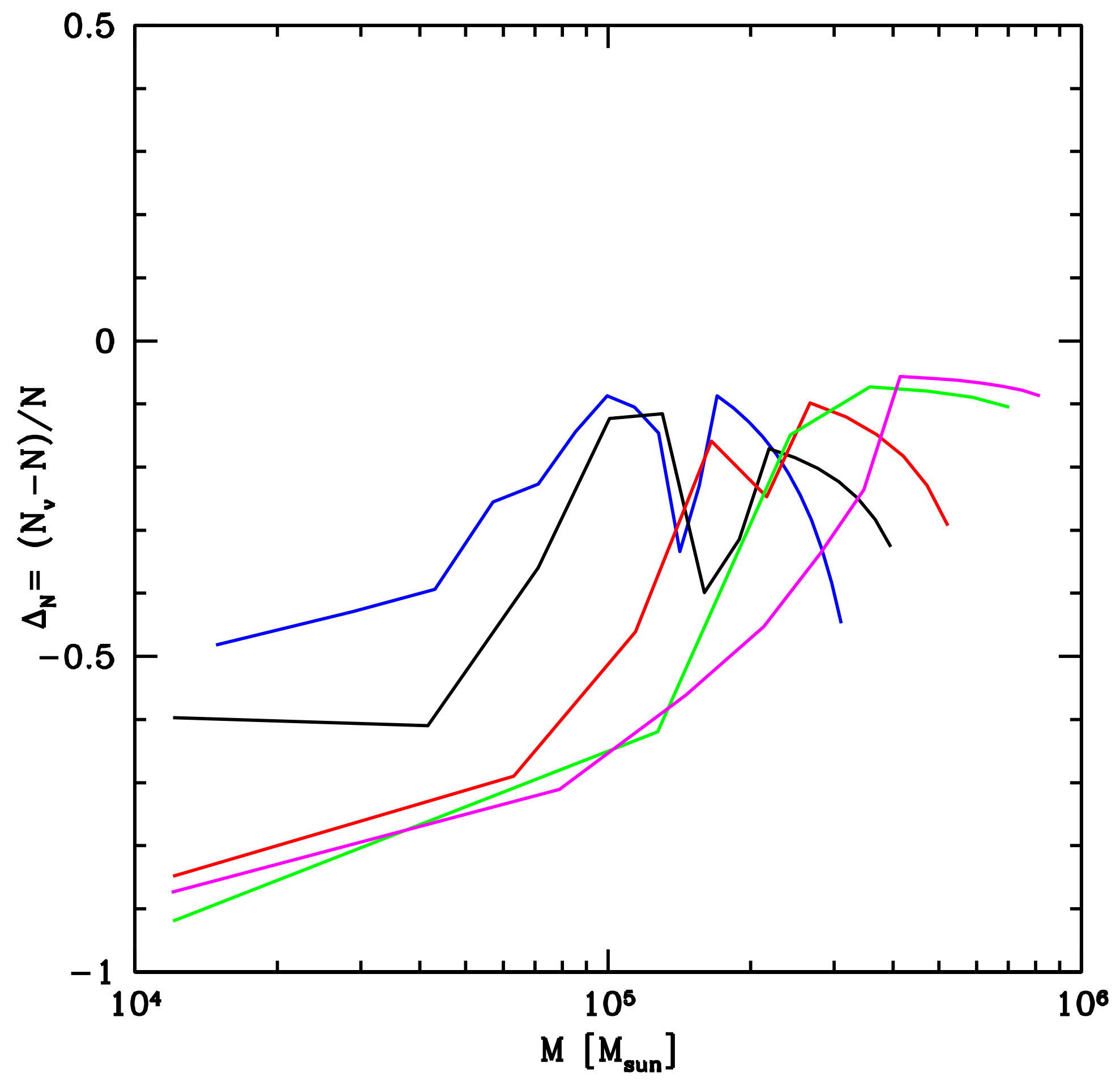




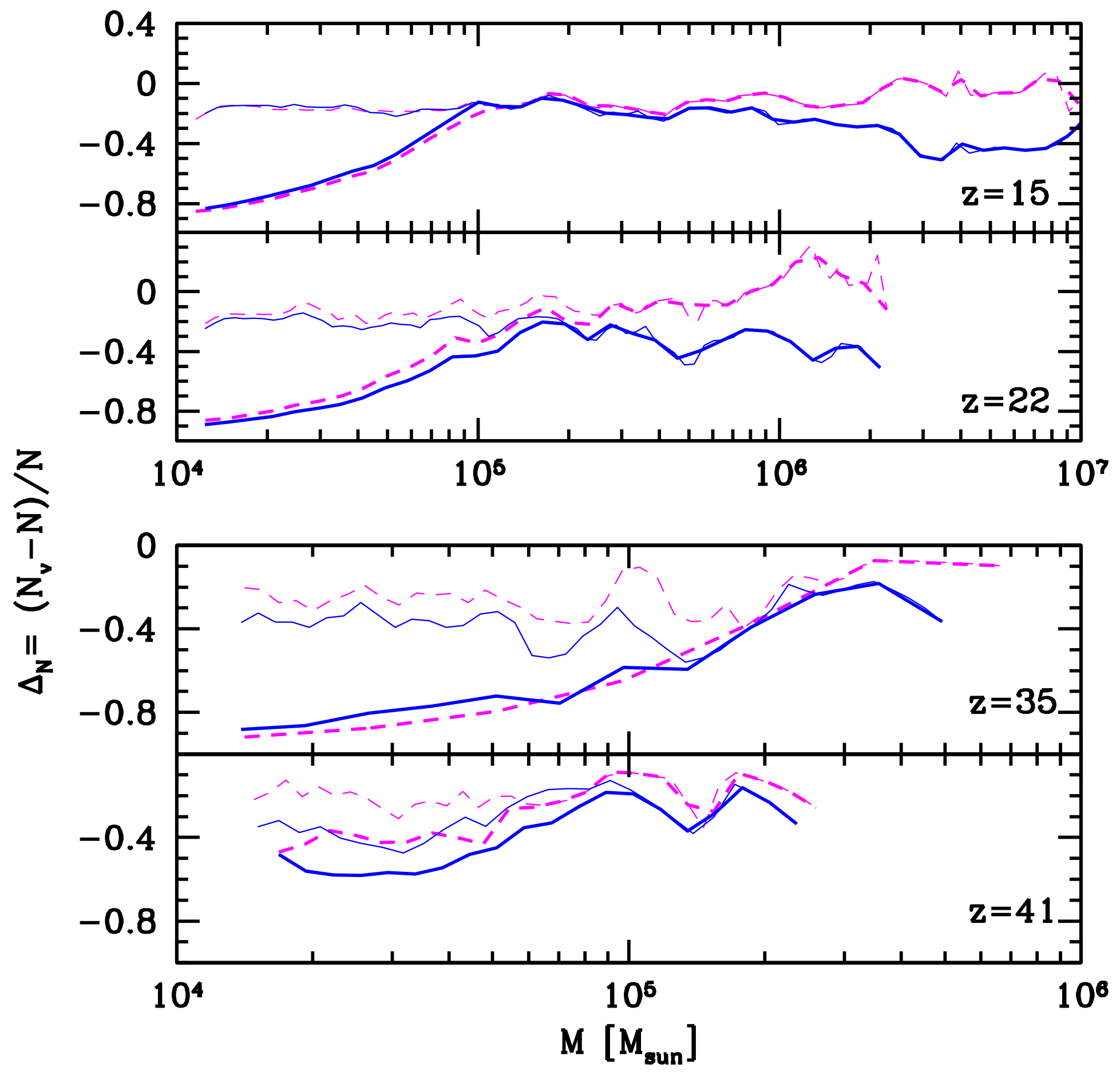

